\title{
28 Research Square \\ Cost-Utility Analysis of Expanding Hepatitis B Vaccination Among Healthcare Workforce in Ethiopia
}

\section{Dinksew Tewihubo}

Medda Wolabu University

Micheale Gebrehans

Mekele University

Getahun Asmamaw ( $\sim$ getahun.asmamaw@amu.edu.et )

Arba Minch University

\section{Research Article}

Keywords: Cost-Utility, Hepatitis-B virus prevalence, Hepatitis-B vaccination coverage, Healthcare Workforce, Ethiopia

Posted Date: July 19th, 2021

DOl: https://doi.org/10.21203/rs.3.rs-239064/v2

License: (c) (i) This work is licensed under a Creative Commons Attribution 4.0 International License. Read Full License 


\section{Abstract}

Objective Ethiopia is one of the countries with high endemicity of hepatitis B infection. In Ethiopia, the current vaccine coverage among health care workers accounts for around $14 \%$. Most health workforce $(241,250)$ of Ethiopia was first considered as susceptible with a probability of getting Hepatitis B Virus acutely and $5-10 \%$ chance of progressing to chronic Hepatitis B. Hence, examining cost-utility analysis of hepatitis $B$ vaccination coverage among healthcare workers in Ethiopia was found the most essential work.

Method Markov model for expanding vaccination coverage ( 3 doses of hepatitis B vaccine) was simulated based on the data obtained both primary and secondary data. A secondary data particularly cost and effectiveness data were obtained from published articles, World Health Organization (WHO) guidelines and Ethiopian Federal Ministry of Health $(\mathrm{FMOH})$ documents. Moreover, cost related data for vaccination and chronic hepatitis $B$ treatment were also gathered by interviewing expertise from Tikur Anbesa Specialized Hospital (TASH). This study was conducted from a healthcare payer perspective, with $3 \%$ discount rate of cost and health outcome as WHO recommendation. Primarily health outcome was measured by Quality Adjusted Life Year (QALY) gain and Incremental Cost-Effectiveness Ratio (ICER). Deterministic analysis and tornado diagrams were employed to manage parameter uncertainty and show a plausible range of cost and effectiveness of variables.

Result Current vaccination program is more expensive (USD 29.99) with a positive incremental cost of USD 1.32 and less effective that have negative incremental effectiveness of -0.08 and total life year gains of 28.54 than Expanded Hepatitis B vaccination strategy which costs USD 28.67 and gives relatively high total life-year gain of 28.62. The resulting ICER was USD 16.23 per QALY gained. However, the ICER was a negative for the current vaccination strategy that could show, it was dominated by the Expanded Hepatitis B vaccination strategy. One-way sensitivity analysis also provided that the current vaccine coverage was dominated for an increase in the risk of infection among unvaccinated individuals.

Conclusion Increasing current vaccine coverage from $14 \%$ to no less than $80 \%$ across Ethiopian healthcare workforces would be the most cost-effective strategy.

\section{Introduction}

Hepatitis B Virus (HBV) is one of the serious global health burdens that affect the lives of more than two billion peoples. Hepatitis B (HepB) is primarily transmitted by percutaneous or mucosal contact of infected blood and body fluids, and occupationally exposure of healthcare workers during dental, medical and surgical procedures ${ }^{1}$. HepB virus could be acute or chronic based on the persistence of HepB surface antigen (HBsAg) for less or more than six months. Most of the acute HepB infections were self-limiting that doesn't require specific treatment. However, $0.5-1 \%$ of individuals may develop fulminant hepatitis with a mortality rate of $70-80 \%$. Meanwhile, $3-5 \%$ of adults with acute HepB have a chance of progressing into chronic $\mathrm{HepB}^{2}$. Chronic HepB could be treated by highly effective antiviral therapy like 
Tenofovir, lamivudine, etc. Despite that only $5 \%$ of Chronic Hepatitis B (CHB) patients have access to treatment for various reasons ${ }^{3}$. Patients with chronic HepB may have a $40 \%$ risk of developing sequelae like cirrhosis, liver failure, and Hepatocellular Carcinoma. Consequently, $15-25 \%$ of peoples with chronic HepB infection die every year due to liver complications ${ }^{3}$.

Recently it was estimated that around 350 million peoples all over the world live as chronic carriers of the virus with a prevalence of $3.5 \%{ }^{4}$. However, the endemicity of HBV varies across the world with the highest prevalence occurring in Asia and Africa ${ }^{5}$. Particularly healthcare workers have 10 times more risk of acquiring HBV infection than the general population ${ }^{6}$. Globally, the annual incidences of hepatitis B infection in healthcare professionals reach about $6 \%{ }^{4}$. Likewise, in Ethiopia, the high risk of exposure was also reported among physicians, clinical nurses, midwives, laboratory technicians, and anesthetists consecutively ${ }^{7}$.

Currently, three doses of monovalent $\mathrm{HepB}$ vaccinations which are given for six months were the most effective and safe way to prevent $\mathrm{HepB}$ infection ${ }^{8}$. Both WHO and Ethiopian $\mathrm{FMOH}$ recommends that all health professionals should be vaccinated against HBV. Those institutions indorsed to reach the HBV vaccination coverage at least $90 \%$ among highly susceptible health professionals such as midwifery, surgeons, dialysis workers, and laboratory reach ${ }^{9}$.

However, across several countries, HBV vaccination coverage among all healthcare workforce who directly or indirectly participate in the healthcare service delivery including health extension workers, medical waste handlers, janitors remained low $(<20 \%)^{10}$. In Ethiopia, the minimal $(14 \%)^{11,12}$ coverage of HepB vaccination among healthcare professionals was also reported. Different studies stated several reasons such as lack of vaccine availability at healthcare centers, higher cost of vaccine at private health facilities, lack of knowledge about the severity of HBV infection, and low attitude towards risk factors and efficacy of the vaccine ${ }^{11,12}$ for the low coverage in the country. Hence, this cost-utility analysis determined the cost of increasing HepB vaccine coverage among all healthcare workforces such as nurses, laboratory, pharmacy, medical doctors, midwives, janitor workers, supportive staff and its effectiveness on disease prevention, QALY gained in comparison to current HepB vaccination coverage.

\section{Method}

Cost-utility analysis of expanding HepB vaccination was conducted in comparison to current Ethiopian HepB coverage among healthcare workers in Ethiopia. Ethiopian $\mathrm{FMOH}$ is the main public healthcare deliverer which is responsible for policy formulation, planning, development, and management of all health issues. The minster office has above 241,250 health workforces under various categories such as health extension workers (15\%), paramedical professionals (35\%), medical doctor professionals (3\%), supportive and administrative staff $(30 \%)$ and others ${ }^{9}$. The average age of individuals was estimated at 32 years. All healthcare workforces in Ethiopia were included in this study. It is because these peoples have a relatively high occupational exposure to the HepB virus as compared to the general population ${ }^{7}$. 
Accordingly, this study was implemented across all health facilities in the country i.e., 16,243 health posts, 3,743 health centers, 220 primary hospitals, 64 general hospitals, 27 referral hospitals, and other administrative offices like the regional health bureau, district health offices, and $\mathrm{FMOH}^{9}$.

The study was conducted from the healthcare payer perspective i.e., Ethiopian $\mathrm{FMOH}$, though vaccines are financed by international donors (Table 1). Finally, the cost of increasing vaccination coverage, effect on the life year gains, quality of life, ICER, and disease prevention of HepB vaccine was evaluated over the lifetime of healthcare workers i.e., 65 years since it was assumed that vaccines provide lifetime protection. The cost and QALY were adjusted to a $3 \%$ discount rate as of WHO recommendation (Table 1). Synthesis based estimate of data was used accordingly, several studies conducted in China, USA, and Ethiopia were referred, and mean value was taken to overcome misleading data findings from a single study.

Table 1

Method summary of economic evaluations of cost-utility analysis of expanding hepatitisB vaccination among healthcare workforce in Ethiopia

\begin{tabular}{|ll|}
\hline Disease & Hepatitis B \\
\hline Population & Healthcare workforce \\
\hline Intervention & Increasing vaccination \\
\hline Comparators & Current vaccination \\
\hline Outcome & Disease prevented, QALY gained, Cost \\
\hline Perspective & Health care payer \\
\hline Time Horizon & Lifetime \\
\hline Discounting & $3 \%$ \\
\hline Sensitivity Analysis & Deterministic Sensitivity analysis \\
\hline Type of Model & Markov modeling \\
\hline
\end{tabular}

All necessary pharmaceuticals including the HepB vaccine were procured and supplied primarily by the Ethiopian pharmaceutical supply agency (EPSA). Antiviral drugs like Tenofovir 300mg, Lamivudine $150 \mathrm{mg}$ alone which are used for the treatment of chronic HepB are not included in the pharmaceutical procurement list of Ethiopia ${ }^{13}$. However, they are assumed to be supplied by EPSA and currently available at a TASH. Cost related data were gathered by interviewing expertise from TASH (Nurses, Physicians, and Pharmacists), WHO HBV guideline. Furthermore, effectiveness related information were also gathered from distinctive pieces of literature review. All costs were expressed in USD at an exchange rate of 28 Ethiopian Birr to USD I on June, 11, 2019. 


\section{Model structure}

As the model of HepB virus disease progress depicted in (Fig. 1) indicates any susceptible individual may stay at "susceptible state" without acquiring an infection. Otherwise, get infected by HBV first acutely with a $5-10 \%$ chance of progressing to chronic HepB and $0.5-1 \%$ case fatality rate. The other chance of susceptible individuals is either develop self-immunity or get vaccinated from prior infection. Any person who has immunity could stay an "immune" health state. The other health state is the "Chronic hepatitis" state. In this health state, an infected person may live as a chronic carrier or get treatment then progress to a healthy ("immune") state. On the other hand, when any abnormality following or resulting from a chronic HepB infection such as liver permanent damage (cirrhosis), the individual inters to "Sequelae" health state. Despite this, any health state has a probability of entering into an absorbing state i.e., death.

\section{Assumptions}

Hepatitis B vaccines are available in different formulations for an instant in combination with other vaccines, like diphtheria-tetanus-pertussis (DTP), Haemophilus influenzae type b (Hib), and Inactivated Poliovirus (IPV). However, in this economic evaluation, only the monovalent HepB vaccine was considered since it is presently implemented with an effectiveness/protection rate of $90 \%$ in Ethiopia 4,9 . The term immunized is considered as healthcare workers receive a complete series of 3 doses of HepB vaccine within a six-month duration. All Healthcare workers who didn't receive the vaccination before were assumed to be eligible for vaccination without being screened for HBV. Utility value for chronic HepB, sequelae, immune and susceptible was adopted from a study conducted in the USA. The transmission of HepB virus infection at health facilities and healthcare workers was not considered in our model. In this economic evaluation treatment cost of acute hepatitis is not included because it is essentially self-limiting infection. For patients with liver complications such as hepatocellular carcinoma and cirrhosis only supportive care like resuscitation, symptomatic treatment was taken into consideration. Vaccination results in life long (up to $65 \mathrm{ELY}$ ) immunization of healthcare workers.

\section{Model Input}

\section{Effectiveness Data}

In this study, the data regarding the chronic HepB disease progression, the natural history of the disease, vaccine effectiveness in infection prevention, treatment effectiveness in reducing disease progression

towards sequelae, and the health outcome was taken from various kinds of literature and guidelines $2,3,14$ as indicated in Table 2. 
Table 2

Various probability estimation of Hepatitis B disease progression obtained from published follow-up studies.

\begin{tabular}{|c|c|c|c|}
\hline Health event incidence/transition & Probability & Range & $\begin{array}{l}\text { Data } \\
\text { source }\end{array}$ \\
\hline $\begin{array}{l}\text { Incidence of hepatitis B infection without vaccination among } \\
\text { healthcare workers }\end{array}$ & 0.05 & $0.4-0.8$ & 16 \\
\hline The transition of acute hepatitis B to chronic hepatitis B & 0.05 & $\begin{array}{l}0.05- \\
0.1\end{array}$ & 2 \\
\hline Progression of Untreated hepatitis B to sequelae (cirrhosis, HCC) & 0.05 & $\begin{array}{l}0.02- \\
0.054\end{array}$ & 1 \\
\hline Death due to hepatitis B infection & 0.0106 & $\begin{array}{l}0- \\
0.0106\end{array}$ & \\
\hline Incidence of sequelae chronic following hepatitis B treatment & 0.0175 & $\begin{array}{l}0.017- \\
0.022\end{array}$ & 2,4 \\
\hline Death due to Hepatitis B complication & 0.2 & $\begin{array}{l}0.15- \\
0.25\end{array}$ & 17 \\
\hline Utility of chronic hepatitis B & 0.87 & - & 15 \\
\hline Utility of sequalae hepatitis B & 0.57 & - & \\
\hline Utility of acute hepatitis B & 0.43 & - & \\
\hline
\end{tabular}

\section{Cost Data}

Cost of three series doses of HepB vaccine (Table 3) for every unvaccinated susceptible health workforce which accounts around $86 \%$ of the currently active healthcare workforce was determined and the price of one dose monovalent HepB vaccine is USD 17.8 and costs related to vaccine administration were also considered (total cost of the vaccine and its administration per cycle length/1 year was estimated at USD 57). For HepB treatment generic TDF 300 mg tablet was given as once-daily dose also optionally Propranolol $1 \mathrm{Omg}$, Furosemide $20 \mathrm{mg}$ tablet may be given as prophylaxis to sequelae and its cost was estimated USD 57 per length cycle (I year) ${ }^{2}$. 
Table 3

Estimated individual annual hepatitis B vaccination cost vs hepatitis B disease management cost

Service List of items consumed/service provided

\begin{tabular}{lll} 
Vaccination & Hepatitis B, glove, syringe & USD 57 \\
\hline Hepatitis B treatment & TDF 300mg daily +/- diuretics & USD 57 \\
\hline Laboratory investigation & HBsAg ALT, AST, INR, BUN, Albumin, Creatinine & USD 88.56 \\
\hline Hospitalization cost & Symptomatic treatment and bed admission & USD 189
\end{tabular}

ALT: Alanine Amino Transferase; AST: Aspartate Amino Transferase; HBsAg: Hepatitis B Surface Antigen; INR: International Normalized Ratio; TDF: Tenofovir

Laboratory monitoring and investigation for a patient with chronic HepB was estimated to cost USD 57. This cost covers most of necessary laboratory tests like liver function tests (ALT, AST), HBsAg, Albumin, $B I R$, and BUN except HBV DNA test which is the most expensive and inaccessible test. For patients who develop sequelae like cirrhosis, Ascites, portal hypertension, and hepatocellular carcinoma cost of hospitalization and symptomatic treatment (USD 189) were also added to laboratory cost and treatment cost. Liver transplant cost for patients with liver failure was not estimated since it is not applicable in Ethiopia.

Most of the information regarding the cost of HepB vaccination and chronic HepB disease management was obtained from pharmacists, nurses, and physicians who actively work in TASH at the department of Gastroenterology, pharmacy, and internal medicine. Indirect cost, transportation cost, capital item costs like cold chain storage, deep freezer vans or vehicles, and other operational costs related to logistics were not included. Finally, the upfront cost for (e.g., fryers/advertisement) to increase vaccine coverage was not considered due to a lack of data (Table 3). The cost of the three series of vaccine doses and its administration costs (one-time costs) were considered at the beginning cycle of the simulation. Whereas the other costs were considered over the life time of health care work forces i.e., 65 years (33 cycles).

\section{Model Output}

An output of this economic evaluation were incremental cost, incremental effectiveness, and qualityadjusted life years gains of expanding HepB vaccination (from 14-80\%) among all health workforce; and finally, its ICER per QALY was calculated.

\section{Sensitivity Analysis}

Deterministic sensitivity analysis was employed to manage some uncertainty relating to methodological assumption (such as time, cost, and effectiveness) variation of distinctive parameters and heterogeneity among healthcare workforce. Additionally, tornado diagram was also used to illustrate the plausible ranges of parameters obtained from published literature and guidelines of the country. 


\section{Results}

The current vaccination coverage (14\%) program is more expensive (USD 29.99) with a positive incremental cost of USD 1.32. It was also less effective having a negative incremental effectiveness of -0.08 and total life year gains of 28.54 than Expanded Hepatitis B vaccination strategy ( $80 \%$ ). Expanding Hepatitis B vaccination costs USD 28.67 that gives relatively high total life-year gain of 28.62. The resulting ICER was USD 16.23 per QALY gained. Overall current vaccination strategy was dominated by increasing vaccination coverage to $80 \%$ (Table 4). The final average costs of the two alternatives were less than the estimated costs at the beginning of the cycle. This is for the reason that all healthcare workers might not get treatment as it could determine by the probability of getting the treatments such as, Tenofovir (due to unavailability and/or expensiveness issues).

Table 4

Base case results from cost-effectiveness analysis of Expanding Hepatitis B vaccination coverage versus Current vaccination coverage (June 2019)

\begin{tabular}{|lccccc|}
\hline Strategy & Cost & Life Years & ICER & NMB & C/E \\
\hline Expanding Hep B vaccination coverage & 28.67 & 28.62 & - & $24,954.85$ & 1 \\
\hline Current He B vaccination coverage & 29.99 & 28.54 & -16.23 & $24,882.53$ & 1.05 \\
\hline ICER: Incremental Cost-Effectiveness Ratio; NMB: Net Monetary Benefits; C/E: Cost Per Effectiveness \\
\hline
\end{tabular}

Since Ethiopia does not have a standardized cost-effectiveness threshold (CET), the World Bank report of Ethiopia's GDP per capita (USD 783) was used to determine CET. Accordingly, GDP was multiplied by three to obtain an estimated CET of USD 2,349 per QALY gains. Despite ICER showed negative, the current vaccination strategy was dominated by increasing vaccination strategy (Fig. 2). Since an increasing in vaccination coverage on cost-effectiveness plane lies on quadrant two. Which made it a less expensive and more effective quadrant.

One-way sensitivity analysis provides that the current vaccine coverage was dominated for an increase in the risk of infection among unvaccinated individuals. For instance, when the probability of infection is increased among unvaccinated individuals; the outcome of ICER has increased from -0.9 to -0.81 . Accordingly, the cost has increased from 1.54 to 2.04 while the effectiveness had decreased from -0.09 to -0.13 as the chance of infection is raised with the current vaccine coverage. The additional incurred cost was related to treatment costs after infection. Similarly, tornado result revealed that the probability of die due to sequelae, death due to hepatitis $B$ infection, the progression of untreated chronic hepatitis $B$ to sequelae, cost of lab investigation, probability of treating chronic hepatitis B is the main parameter which possibly affects the ICER value (Fig. 3). Varying cost parameters in the allowable range could increase the ICER value and the rest of the parameters might reduce or increase the ICER value base on the range. 
From the two-way sensitivity analysis (Fig. 4), it was found that irrespective of probability of death occurrence in chronic hepatitis $B$ and the probability of $\mathrm{HBV}$ infection in unvaccinated individuals increasing vaccine coverage improve total QALY gain.

\section{Discussion}

The foremost aim of this economic evaluation was to compare cost-utility analysis of increasing vaccination by $80 \%$ versus keeping current coverage (14\%). By doing these costs required per every healthcare worker in either strategy was determined, welfare obtained in kind of life year gains, utility increment and infection prevention were also calculated. Several secondary data were used to conduct this analysis that obtained from published articles particularly effectiveness data. Moreover, estimated cost data for vaccination and chronic hepatitis B treatment was also collected from TASH ${ }^{1,3,15}$.

The new expanded coverage (80\%) strategy reduces the cost by USD 16.23 that was expended by the current vaccination program for every QALY gains in lifetime horizon. Besides this promoting vaccination coverage was necessary as it is a less expensive and highly effective strategy to combat hepatitis $B$ infection among healthcare workers. Alongside in Ethiopian health care system economic evaluations was not common practice, hence conducting such analysis would help policymakers, public healthcare payers to invest scarce healthcare resource cost-effectively.

Deterministic sensitivity analyses showed that varying the probability of different variables of cost and effectiveness as the new strategy significantly dominates the current program (Fig. 2). While tornado result indicates that some variables impact on ICER value (Fig. 3). Cost-effectiveness threshold of USD 2,349 i.e., three times of Ethiopia GDP was used to check the affordability of the new strategy. Therefore, since the ICER has a negative value implementation of the new program could not affected by the cost of the program.

In general, increasing vaccination coverage by $80 \%$ among healthcare work forces was found costeffective in Ethiopia compared to the current coverage (14\%) alternative. Furthermore, the Hep B monovalent vaccine highly effective in preventing the infection which gives a $90 \%$ effectiveness on the life time protection of the healthcare workers ${ }^{8}$. Hence; with a high risk of infection and burden of Hep B among healthcare workers and minimal reduction of the prevalence with the current coverage (from 7.4$6 \%)^{16}$, the new strategy is highly recommended in Ethiopia as it was more effective and saves additional cost that would be consumed by the current coverage (14\%) strategy. Existing evidences also supports the high prevalence of Hep B among healthcare works Ethiopia. For instance, the seroprevalence of Hepatitis-B viral infection among the sample healthcare workers was $4.52 \%(95 \% \mathrm{Cl}: 2.4,6.5)$ and $2.6 \%$ at

Gondar and Addis Ababa referral hospitals respectively ${ }^{7,12}$. Moreover, the pooled estimation of prevalence among healthcare work presented a $5 \%$ prevalence in Ethiopia. When compared with other studies from African countries, the burden of Hep B viral infection is somewhat lesser. Health works are at high risk for viral infection compared to other ordinary people. Predisposing factors such as contamination due to medical procedures, workover load, and other factors are claimed to be responsible 11,12,14,16. Moreover, 
regarding the accessibility of vaccines for health work remind a challenge particularly unavailability and cost are repotted ${ }^{11,12,14}$. This implies the burden of this viral infection is being an additional challenge for the health system of Ethiopia since the government is facing other diseases such as infectious and noncommunicable diseases.

\section{Study limitation}

This economic evaluation has some limitations especially regarding costing variables. We did not include viral transmission rates among study participants, this might underestimate the benefit. Indirect cost related to increasing vaccination coverage such as vaccine transport and cold chain equipment cost was not included even though the expansion of vaccination coverage to around $80 \%$ over all the country health facility requires a high investment of capital items. This might cause an underestimation cost of the expanded vaccination program and might result in the absolute dominance of the current vaccination strategy. So, we recommend for future studies to include those costs.

On the other hand, the cost of a liver transplant for patients with liver failure and the cost of acute hepatitis B treatment was not explicitly determined. Also, direct non-medical cost like transport cost, loss of work due to the disease of every healthcare workforce was also not included. Although this study gives high emphasis to reduce healthcare worker occupational exposure to hepatitis B infection by providing vaccines, it does not determine the cost of screening before vaccination it uses a methodological assumption of all healthcare workers are susceptible and eligible to vaccination. Of despite all these limitations efforts have been made to use reliability data by gathering cost data from active physicians and other health professionals in TASH. In addition, a sensitivity analysis was conducted to test the robustness of the model to check whether it is consistent with the base-case analysis or not.

\section{Conclusion}

From this economic evaluation, it is possible to conclude that increasing current vaccine coverage from $14 \%$ to at least $80 \%$ among all Ethiopian all healthcare workforces was the most cost-effective strategy. As long as expanding hepatitis B vaccine coverage does not incur additional costs and in the meantime, it improves the healthy life of susceptible healthcare workers Ethiopian FMOH should work on its implementation.

\section{Abbreviations}

ALT: Alanine Amino Transferase; AST: Aspartate Amino Transferase; BIR: Bilirubin; BUN: Blood Urea Nitrogen; CHB: Chronic Hepatitis B; DNA: Deoxyribo-Nucleic Acid; ELY: Expected Life Year; EPSA: Ethiopian Pharmaceutical Supply Agency; FMOH: Federal Ministry of Health; GDP: Gross Domestic Product; HBsAg: Hepatitis B Surface Antigen; HBV: Hepatitis B Virus; HCC: Hepato-Cellular Carcinoma; HepB: Hepatitis B; ICER: Incremental Cost-Effectiveness Ratio; INR: International Normalized Ratio; QALY: Quality Adjusted Life Year; USD: United States Dollar; USA: United States of America; WHO: World Health Organization. 


\section{Declarations}

\section{Acknowledgment}

We would like to thank Tikur Anbesa Specialized Hospital (TASH) for their fantastic collaboration to provide dataset.

\section{Authors' contributions}

DT and MG, conceived of research problem and collected the dataset. DT, MG, and GA did a literature review and performed data analysis. GA and DT critically drafted and reviewed the manuscript. The manuscript has read and approved by all authors in this study.

\section{Competing Interests}

None

\section{Funding}

None

\section{Availability of Data and Materials}

All data generated and analyzed during this during study are included in this published article and publicly available.

\section{Ethics Approval and Consent to Participate}

Not Applicable

\section{Consent for Publication}

All authors agreed to publish this research article

\section{References}

1. Kwon SY, Lee CH. Epidemiology and prevention of hepatitis B virus infection. Korean J Hepatol. 2011;17(2):87-95. doi:10.3350/kjhep.2011.17.2.87

2. World Health Organization. World Health Organisation. Guidelines for the Prevention, Care and Treatment of Persons with Chronic Hepatitis B Infection. 2015. Geneva. World Health Organisation.; 2015. http://apps.who.int/iris/bitstream/10665/154590/1/9789241549059_eng.pdf?ua=1\&ua=1.

3. Chang MS, Nguyen MH. Epidemiology of hepatitis B and the role of vaccination. Best Pract Res Clin Gastroenterol. 2017;31(3):239-247. doi:10.1016/j.bpg.2017.05.008 
4. WHO. Position paper on Hepatitis B. Who. http://www.who.int/entity/wer/2009/wer8440.pdf. Published 2017.

5. Schweitzer A, Horn J, Mikolajczyk RT, Krause G, Ott JJ. Estimations of worldwide prevalence of chronic hepatitis B virus infection: A systematic review of data published between 1965 and 2013. Lancet. 2015;386(10003):1546-1555. doi:10.1016/S0140-6736(15)61412-X

6. Nigussie ZY, Tafere TE, Kassa GT. Managements of Exposure to Blood and Body Fluids Among Healthcare Workers and Medical Students in University of Gondar Hospital, Northwest of Ethiopia. Glob J Med Res Interdiscip. 2013;13(5):1-9.

7. Gebremariam AA, Tsegaye AT, Shiferaw YF, Reta MM, Getaneh A. Seroprevalence of Hepatitis B Virus and Associated Factors among Health Professionals in University of Gondar Hospital, Northwest Ethiopia. Adv Prev Med. 2019;2019:1-5. doi:10.1155/2019/7136763

8. Ott JJ, Stevens GA, Groeger J, Wiersma ST. Global epidemiology of hepatitis B virus infection: New estimates of age-specific HBsAg seroprevalence and endemicity. Vaccine. 2012;30(12):2212-2219. doi:10.1016/j.vaccine.2011.12.116

9. FDRE $(\mathrm{MOH})$. National hlealth Workforce Update Development Directorate Bulletin 2nd Edition. In: ; 2019. http://www.moh.gov.et/ejcc/en/human-resource.

10. Schillie S, Murphy T V., Sawyer M, et al. CDC Guidance for Evaluating Health-Care Personnel for Hepatitis B Virus Protection and for Administering Postexposure Management. Vol 62.; 2013.

11. Abeje G, Azage M. Hepatitis B vaccine knowledge and vaccination status among health care workers of Bahir Dar City Administration, Northwest Ethiopia: a cross sectional study. BMC Infect Dis. 2015;15(1):30. doi:10.1186/s12879-015-0756-8

12. Gizachew Taddesse Akalu, Addis Tamire Woldemariam, Abate Bane Shewaye DAG, Abebe Habteselasie Demise, Melaku Tesfaye Debele KDT. Burden of hepatitis-B infections and risk factors among healthcare workers in resource limited setting, Addis Ababa, Ethiopia. EC Microbiol. 2016;4(2016):722-731.

13. PFSA. No Title. https://epsa.gov.et/.

14. Ayalew MB, Horsa BA. Hepatitis B Vaccination Status among Health Care Workers in a Tertiary Hospital in Ethiopia. 2017;2017. doi:10.1155/2017/6470658

15. Jacobs RJ, Saab S, Meyerhoff AS. The cost effectiveness of hepatitis immunization for US college students. J Am Coll Health Assoc. 2003;51(6):227-236. doi:10.1080/07448480309596355

16. Yazie TD, Tebeje MG. An updated systematic review and meta-analysis of the prevalence of hepatitis B virus in Ethiopia. BMC Infect Dis. 2019;19(1):917. doi:10.1186/s12879-019-4486-1 
17. Nelson NP, Easterbrook PJ, McMahon BJ. Epidemiology of Hepatitis B Virus Infection and Impact of Vaccination on Disease. Clin Liver Dis. 2016;20(4):607-628. doi:10.1016/j.cld.2016.06.006

Figures

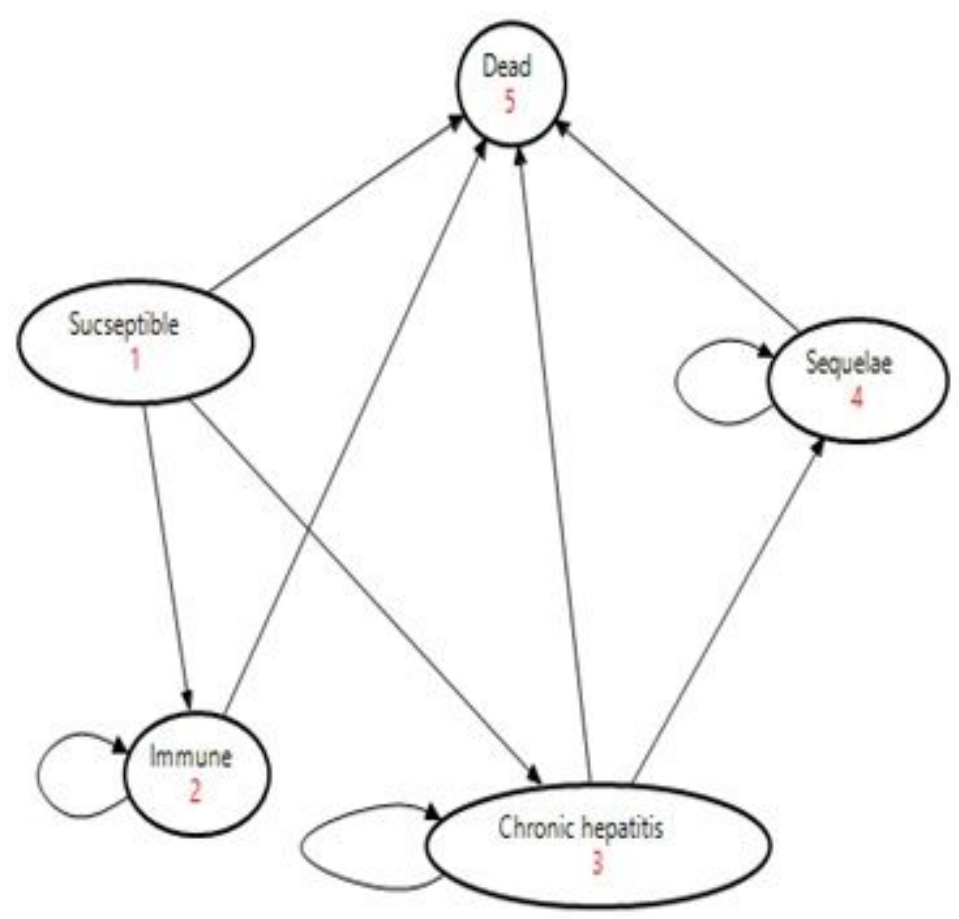

Figure 1

Model of Hepatitis B virus disease progress and consequence 


\section{Cost-Effectiveness Analysis}

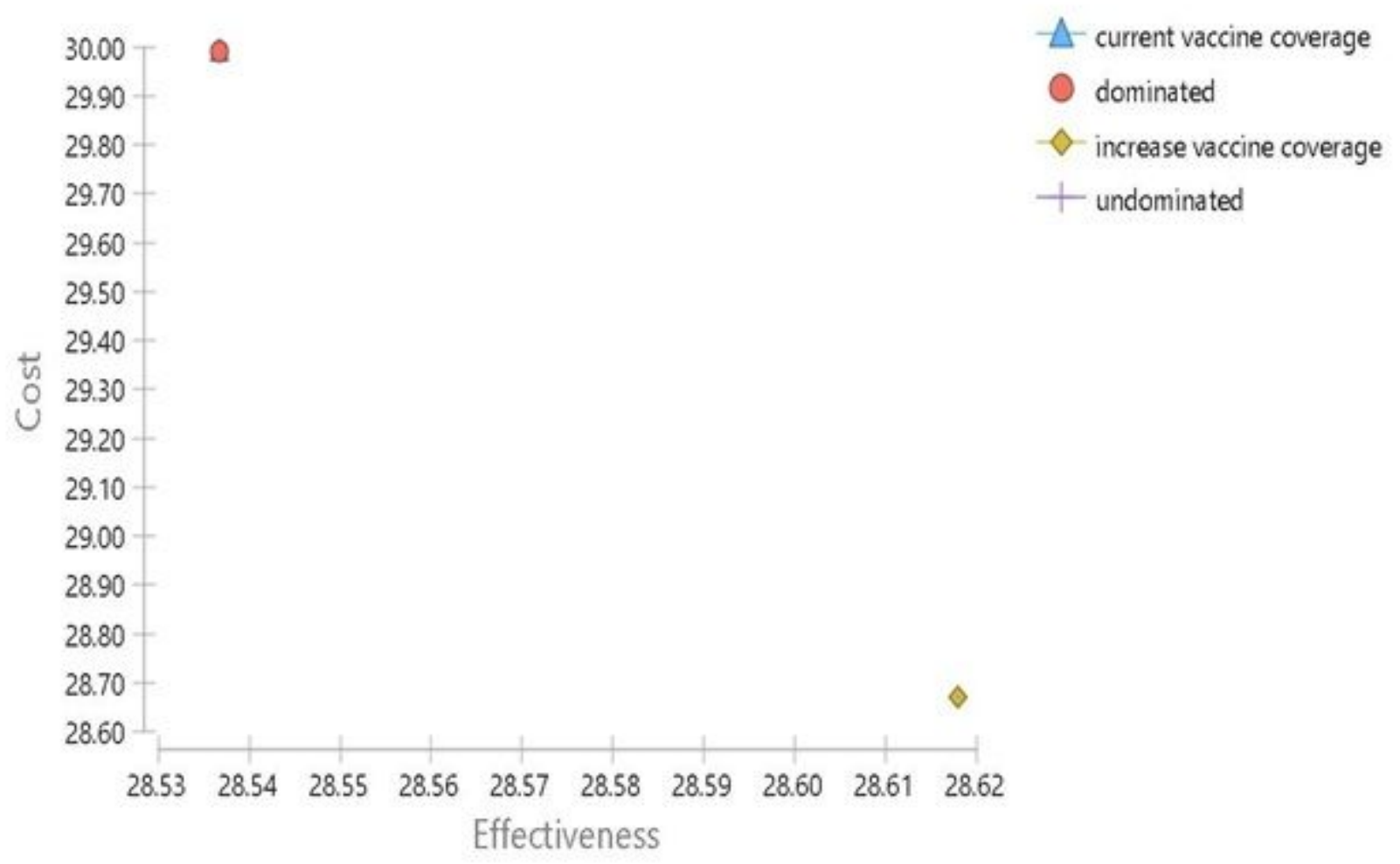

Figure 2

Cost-effectiveness analysis of increasing Hepatitis B vaccination coverage 


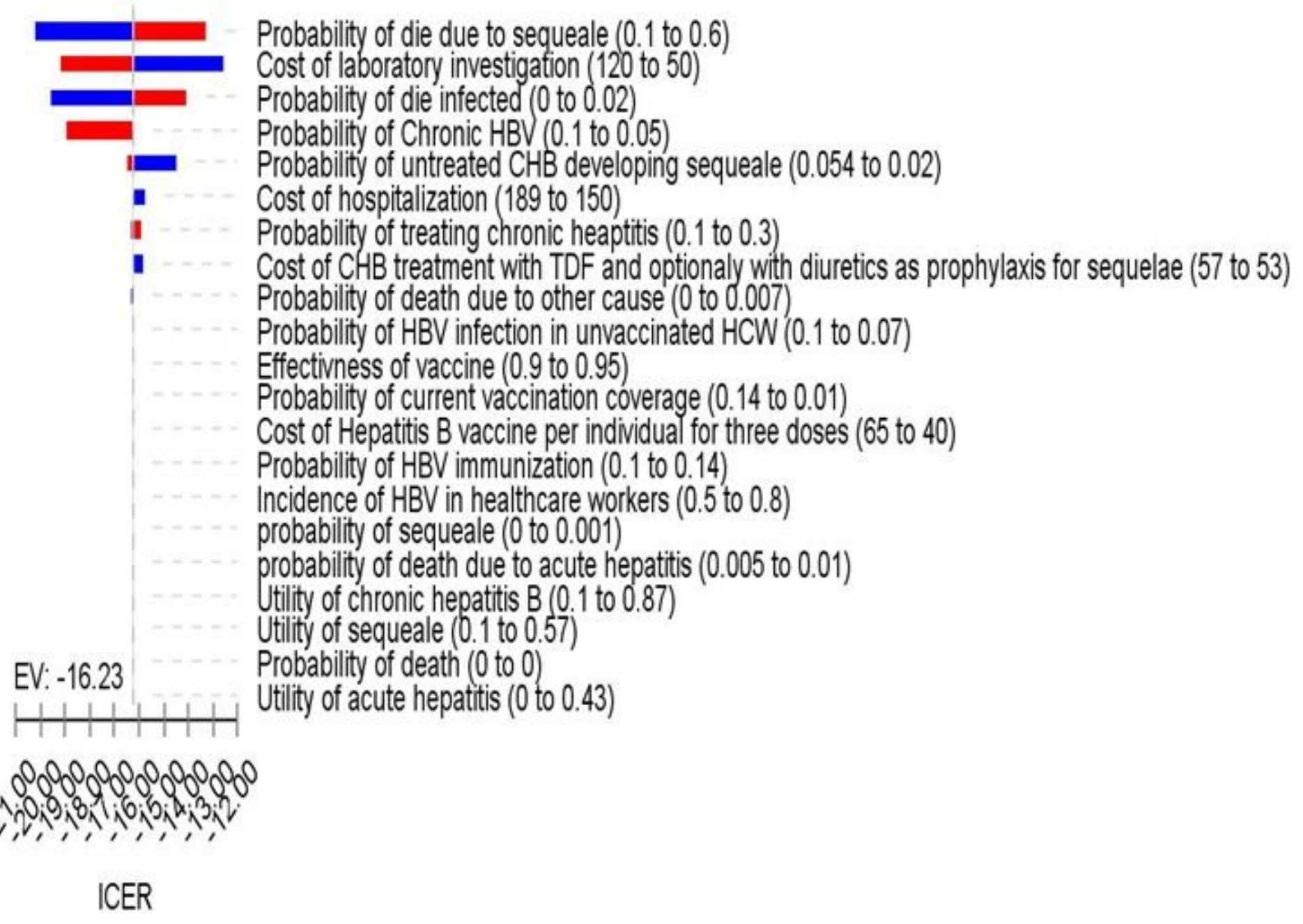

\section{Figure 3}

Tornado diagram of current hepatitis coverage versus increasing current hepatitis coverage (Net Benefit, WTP=873.0)

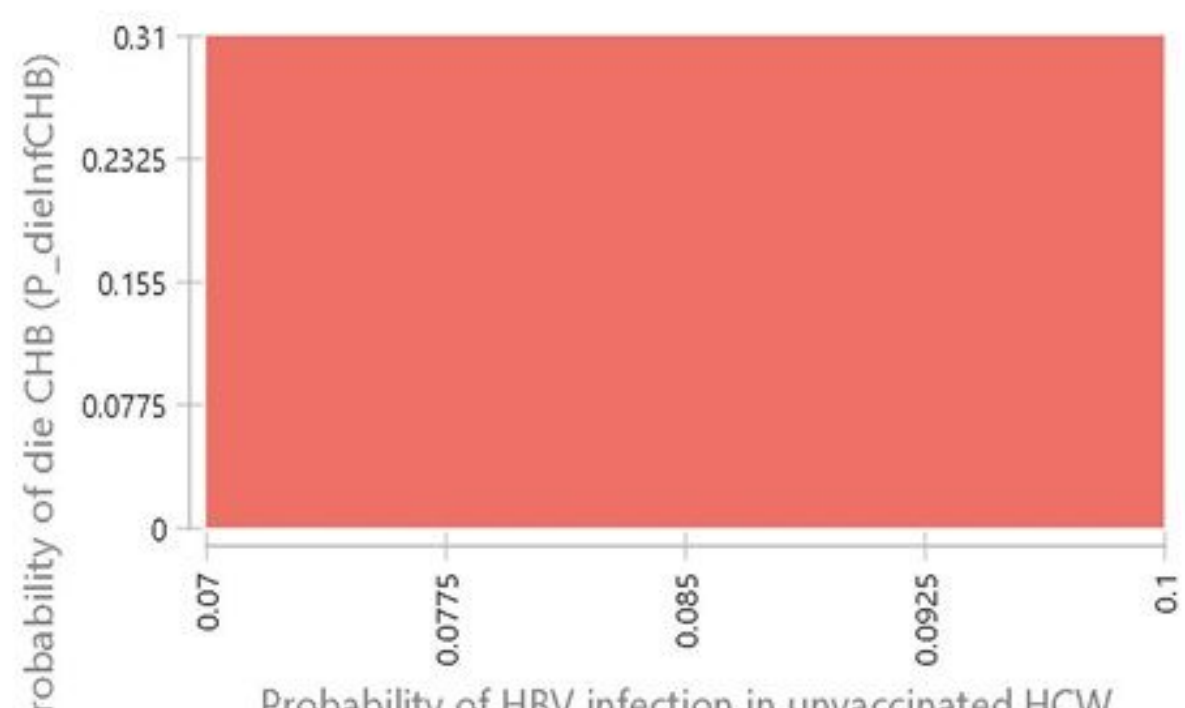

current vaccine coverage

increase vaccine coverage

Probability of $\mathrm{HBV}$ infection in unvaccinated HCW 
Figure 4

Two-way sensitivity analysis of infection among unvaccinated and death due CHB infection 